\title{
BOUNDARY-VALUE PROBLEMS FOR THE INHOMOGENEOUS SCHRÖDINGER EQUATION WITH VARIATIONS OF ITS POTENTIAL ON NON-COMPACT RIEMANNIAN MANIFOLDS
}

\begin{abstract}
We study solutions of the inhomogeneous Schrödinger equation $\Delta u-c(x) u=g(x)$, where $c(x), g(x)$ are Hölder functions, with variations of its potential $c(x) \geqslant 0$ on a noncompact Riemannian manifold $M$. Our technique essentially relies on an approach from the papers by E. A. Mazepa and S. A. Korol'kov connected with introduction of equivalency classes of functions. It made it possible to formulate boundary-value problems on $M$ independently from a natural geometric compactification. In the present work, we obtain conditions under which the solvability of boundary-value problems of the inhomogeneous Schrödinger equation is preserved for some variations of the coefficient $c(x) \geqslant 0$ on $M$.

Key words: inhomogeneous Schrödinger equation, variations of coefficients, boundary-value problems, Riemannian manifold
\end{abstract}

2020 Mathematical Subject Classification: 31C12

1. Introduction. In the studies of recent decades, a strong connection was often noted between the classical problems of function theory and the theory of solutions of elliptic partial differential equations of the second order, in particular, the Laplace-Beltrami equation and the stationary Schrödinger equation. This connection found its development in the works of such Russian and foreign mathematicians as M. Anderson, A. Grigorian, S. Korolkov, V. Kesselman, A. Losev, E. Mazepa, V. Miklyukov, N. Nadirashvili, D. Sullivan, V. Tkachev, and a number of others.

In modern mathematics, the study of elliptic equations on non-compact Riemannian manifolds covers a fairly significant part of the research, the origins of which go back to the classification theory of non-compact Riemann surfaces and manifolds. This theory is based on the study of some

(C) Petrozavodsk State University, 2021 
functional classes on these geometric objects. Sufficiently complete understanding of the history of development and the current state of this scientific field can be obtained, for example, from the paper [3]. An important class of problems of this direction is related to obtaining theorems of the Liouville type, which assert triviality of spaces of bounded solutions of some elliptic equations on a manifold. So, the classical formulation of Liouville's theorem asserts that every bounded harmonic function in $R^{n}$ is constant (two-sided Liouville's theorem). In some studies, there is another approach to Liouville's theorem - it is argued that the space of positive superharmonic functions on a noncompact manifold is trivial (one-sided Liouville's theorem). This property was used as the basis for the concept of the parabolicity of a manifold. Thus, in $R^{n}$ for $n=2$, the one-sided Liouville's theorem holds ( $R^{2}$ is a parabolic manifold), but for $n \geqslant 3$ this does not hold. In works of a number of the authors, the conditions ensuring the validity of the Liouville's theorems of these types on noncompact Riemannian manifolds are adduced in terms of volume growth, or isoperimetric inequalities, or capacity, and so on (see [3], [4], [6]).

However, as was shown in the 80s of the last century, there is a sufficiently large class of manifolds on which nontrivial bounded solutions of elliptic differential equations exist, in particular, the Laplace-Beltrami equation. For example, M. Anderson [1] and D. Sullivan [18] showed that on a simply connected manifold with negative sectional curvature there is an infinite-dimensional set of nontrivial bounded harmonic functions. Moreover, they showed the solvability of the Dirichlet problem on recovering a harmonic function on such manifolds from continuous boundary data at "infinity".

A number of authors (see, for example, [4], [5], [8], [10]) considered solutions of elliptic equations more general than the Laplace-Beltrami equation, on non-compact Riemannian manifolds. For example, the solutions of the stationary Schödinger equation were studied:

$$
L u \equiv \Delta u-c(x) u=0,
$$

where $c(x) \geqslant 0$ is a continuous function on the manifold.

In the paper [4], a certain estimate for the dimension of the space of solutions of this equation was obtained. In the papers [8], [10], various conditions were found for the solvability of boundary-value problems for bounded solutions of equation (1) on non-compact Riemannian manifolds.

For equation (1), it is also possible to formulate both one-sided and 
two-sided Liouville's theorems, taking into account the fact that only the zero constant is a solution to this equation.

An important result was obtained in [5]: the relationship between the validity of the one-sided and two-sided Liouville's theorems and the unique solvability of some exterior boundary problems was studied. In addition, this paper investigates the question of preserving Liouville's property for bounded solutions of the equation (1) for some variations of the coefficient $c(x) \geqslant 0$. The following theorem is proved in [5]:

Theorem. [5] Let $0 \leqslant c_{1}(x) \leqslant A c(x)$, where $A=$ const $>0, \quad c_{1}(x) \not \equiv 0$. If the two-sided Liouville's theorem holds for the equation $\Delta v-c_{1}(x) v=0$, then it also does for the equation $\Delta u-c(x) u=0$.

Both problems - the solvability of the Dirichlet problem and the validity of Liouville's theorem - are quite interesting from the point of view of their study on non-compact Riemannian manifolds. Note that the formulation of boundary-value problems on an arbitrary non-compact Riemannian manifold may be problematic. In some cases, geometric compactification of the manifold makes it possible to formulate problems similarly to the classical Dirichlet problem in bounded domains of $R^{n}$ (for example, [10], [17]).

On the other hand, the paper [14] proposes an approach based on the concept classes of equivalent functions. Later, this approach was developed, for example, in [7], [8]. It made it possible to formulate boundary-value problems on manifolds in the absence of a natural geometric compactification and to study the question of preserving the solvability of boundary-value problems for the equation (1) when the coefficient $c(x)$ varies. In [14], a condition was obtained, under which the solvability of boundary-value problems on the manifold $M$ for the equations $\Delta u-c(x) u=0$ and $\Delta u=0$ implies the solvability of the similar boundary-value problem for equations $\Delta v-c_{1}(x) v=0$ if $0 \leqslant c_{1}(x) \leqslant c(x)$ and $c_{1}(x) \not \equiv 0$.

All the results indicated above consider solutions of linear homogeneous equations on non-compact manifolds without boundary or with compact boundary. Naturally, the questions about formulation and solvability of boundary-value problems for inhomogeneous linear elliptic equations on non-compact Riemannian manifolds arise. In recent years, a sufficient number of papers have appeared on this topic (for example, [9], [12], [13], [16]). In these papers, the asymptotic behavior of solutions of the Poisson equation was studied, as well as the solvability of boundary and exterior 
boundary-value problems for the inhomogeneous Schrödinger equation

$$
L u \equiv \Delta u-c(x) u=g(x),
$$

where $c(x) \geqslant 0$, on non-compact Riemannian manifolds.

The aim of the paper is to obtain conditions under which the solvability of boundary-value problems for the inhomogeneous equation (2) is preserved under variations of the coefficient $c(x)$ on $M$. The research is based on the study of the asymptotic behavior of bounded solutions $u \in C^{2}(M)$ of equation (2) on non-compact Riemannian manifolds $M$ without boundary. In the sequel, we assume that $c(x), g(x) \in C^{0, \alpha}(G)$ for all precompact subset $G \subset M, 0<\alpha<1$.

Note that if $g(x) \equiv 0$, then the equation (2) is the stationary Schrödinger equation, for which this problem has been studied in detail in [14]. Therefore, we assume that $g(x) \not \equiv 0$.

2. The main concepts and auxiliaries. Let $M$ be an arbitrary smooth connected non-compact Riemannian manifold without a boundary, $G \subset M$ be an arbitrary precompact subset with a smooth boundary, $L$ be a second-order linear elliptic operator on $M$ defined in (2). Denote by $\lambda(x), \Lambda(x)$, respectively, the smallest and largest eigenvalues of the operator $L$ in $G$. Recall that if $\lambda \geqslant \lambda_{0}>0$, where $\lambda_{0}$ is a constant, then the operator $L$ is called strictly elliptic in $G$. The operator $L$ is called uniformly elliptic in $G$ if the ratio $\frac{\Lambda(x)}{\lambda(x)}$ is bounded in $G$. It is known that if the coefficients of the operator $L$ are continuous in $G$, then in any subset $G^{\prime} \subset G$ it is a uniformly elliptic operator (see, for example, [2, c. 38-39]).

Compactness lemma. Let $G \subset M$ be a precompact open connected subset on $M$, the operator $L$ be strictly elliptic, and its coefficients belong to the class $C^{0, \alpha}(G)$. Let, also, the family of solutions $\left\{\varphi_{i}\right\}_{i=1}^{\infty}$ of the equation (2) be uniformly bounded on $G$, and $\varphi_{i} \in C^{2, \alpha}(G)$ for any $i$. Then the family of functions $\left\{\varphi_{i}\right\}_{i=1}^{\infty}$ is compact in the class $C^{2}\left(G^{\prime}\right)$ for any compact subset $G^{\prime} \subset G$.

Proof. For each point $x \in M$, there is a neighborhood $O(x) \subset M$, homeomorphic to $R^{n}$. Since $G^{\prime}$ is a compact subset of $G$, there exists a finite number of neighborhoods $O_{s}$, such that

$$
G^{\prime} \subset \bigcup_{s=1}^{m} O_{s} \subset G .
$$

In addition, the operator $L$ is strictly elliptic, and its coefficients belong to the class $C^{0, \alpha}(G)$. Then, for any precompactor subset of $O_{s}^{\prime} \subset O_{s}$, 
such that $\operatorname{dist}\left(O_{s}^{\prime}, \partial O_{s}\right) \geqslant d_{s}>0$ for all functions $\varphi_{i}$, Schauder's internal estimates are fulfilled when $x \neq y([2$, p. 94-95]):

$$
\begin{gathered}
d_{s} \sup _{k} \sup _{O_{s}^{\prime}}\left|\frac{\partial \varphi_{i}}{\partial x_{k}}(x)\right|+d_{s}^{2} \sup _{k, p} \sup _{O_{s}^{\prime}}\left|\frac{\partial^{2} \varphi_{i}}{\partial x_{k} \partial x_{p}}(x)\right|+ \\
+d_{s}^{2+\alpha} \sup _{k, p} \sup _{x, y \in O_{s}^{\prime}} \frac{\left|\frac{\partial^{2} \varphi_{i}}{\partial x_{k} \partial x_{p}}(x)-\frac{\partial^{2} \varphi_{i}}{\partial x_{k} \partial x_{p}}(y)\right|}{|x-y|^{\alpha}} \leqslant \\
\quad \leqslant C_{s}\left(\sup _{O_{s}}\left|\varphi_{i}(x)\right|+\sup _{x, y \in O} \frac{|g(x)-g(y)|}{|x-y|^{\alpha}}\right),
\end{gathered}
$$

where $C_{s}$ is a constant that depends on the properties of the operator $L, n$ and the domain $O_{s}, 0<\alpha<1$.

Note that the sets $O_{s}^{\prime}$ can be chosen so that $G^{\prime} \subset \bigcup_{s=1}^{m} O_{s}^{\prime} \subset G$. Indeed, we take an increasing sequence of open subsets of

$$
O_{s}^{1} \subset \subset O_{s}^{2} \subset \subset \ldots \subset \subset O_{s}
$$

such that $O_{s}=\bigcup_{k=1}^{\infty} O_{s}^{k}$ in each domain $O_{s}$. Then

$$
G^{\prime} \subset \bigcup_{s=1}^{m} O_{s}=\bigcup_{s=1}^{m} \bigcup_{k=1}^{\infty} O_{s}^{k}=\bigcup_{k=1}^{\infty} \bigcup_{s=1}^{m} O_{s}^{k} \subset G
$$

that is, the sets $O^{k}=\bigcup_{s=1}^{m} O_{s}^{k}$ form an open covering of the set $G^{\prime}$. And the following relation holds: $O^{1} \subset \subset O^{2} \subset \subset \ldots \subset \subset \bigcup_{s=1}^{m} O_{s}$. Since $G^{\prime}$ is a compact subset in $G$, we can choose the finite subcovering $O^{1}, O^{2}, \ldots, O^{r}$ from this open covering. Given the increasing sequence $O^{k}$, we get $G^{\prime} \subset O^{r}=\bigcup_{s=1}^{m} O_{s}^{r} \subset G$. So, we can take $O_{s}^{\prime}=O_{s}^{r}$.

Next, select $d=\min _{s=1, \ldots, m} d_{s}, C=\max _{s=1, \ldots, m} C_{s}$. Then on $G^{\prime}$ the Schauder's internal estimates are fulfilled with the constants $d$ and $C$, where $C$ depends on $L, n$, and $G$.

These estimates, given the uniform boundedness of the family of functions $\left\{\varphi_{i}\right\}_{i=1}^{\infty}$ on $G$ and Hölder continuity of function $g(x)$, imply the equicontinuity of this family, as well as the uniform boundedness and equicontinuity of the families of the first and second derivatives of these functions on $G^{\prime}$. Hence, we obtain compactness of this family in the class $C^{2}\left(G^{\prime}\right)$. The lemma is proved. 
Remark 1. The Compactness lemma for the stationary Schrödinger equation was proved in [11].

Further, denote by $\left\{B_{k}\right\}_{k=1}^{\infty}$ the exhaustion of the manifold $M$, i. e., the sequence of open connected nonempty subsets of the Riemannian manifold $M$, such that $\overline{B_{k}} \subset B_{k+1}, M=\bigcup_{k=1}^{\infty} B_{k}$.

Existence lemma. Let $\left\{B_{k}\right\}_{k=1}^{\infty}$ be an exhaustion of $M$ with smooth boundaries $\partial B_{k}$, the operator $L$ be strictly elliptic, and its coefficients belong to the class $C^{0, \alpha}(G)$ for all compact subset $G \subset M$. Let also the family of functions $\left\{\varphi_{k}\right\}_{k=1}^{\infty}$ be uniformly bounded on $M$, and for each $k$ the function $\varphi_{k} \in C^{2, \alpha}\left(B_{k}\right)$ be a solution of the boundary-value problem

$$
L \varphi_{k}=g(x) \quad \text { in } \quad B_{k},\left.\quad \varphi_{k}\right|_{\partial B_{k}}=\left.f\right|_{\partial B_{k}},
$$

where $f$ is some continuous function on $M$. Then this family has a limit function that is a bounded solution of the equation (2) on $M$.

Proof. The uniform boundedness of the family $\left\{\varphi_{k}\right\}_{k=1}^{\infty}$ on $M$ implies its uniform boundedness on the set $B_{k}$ for any $k$, i. e., there exists a constant $C>0$, such that $\left|\varphi_{k}(x)\right| \leqslant C$ for any $k$ and $x \in M$. Next, we apply the Compactness lemma to this family of solutions. Take the sets $B_{k}$ for $k=1,2, \ldots$ for the set $G$. We have the compactness of the family $\left\{\varphi_{k}\right\}_{k=1}^{\infty}$ in the class $C^{2}\left(B_{1}\right)$, which implies the existence of the limit function

$$
\varphi_{f}^{1}=\lim _{k \rightarrow \infty} \varphi_{k}^{1}
$$

on the set $B_{1}$ : it is a solution of the equation (2). Here $\left\{\varphi_{k}^{1}\right\}$ is a convergent subsequence of the sequence $\left\{\varphi_{k}\right\}$. It is clear that $\left|\varphi_{f}^{1}\right| \leqslant C$ on $B_{1}$.

Next, consider the subsequence $\left\{\varphi_{k}^{1}\right\}$ as a sequence of solutions to the equation (2) on the set $B_{2}$. Then there is a limit function

$$
\varphi_{f}^{2}=\lim _{k \rightarrow \infty} \varphi_{k}^{2}
$$

on this set, which is a solution of the equation (2), and $\varphi_{f}^{2}$ is the bounded function on $B_{2}$. Here $\left\{\varphi_{k}^{2}\right\}$ is a convergent subsequence of the sequence $\left\{\varphi_{k}^{1}\right\}$. Moreover, due to the uniqueness of the existence of the limit of a convergent subsequence, the function $\varphi_{f}^{2}$ is the continuation of the function $\varphi_{f}^{1}$ on the set $B_{2}$, i. e., $\left.\varphi_{f}^{2}\right|_{B_{1}}=\varphi_{f}^{1}$.

We continue the process for any $n$ and get existence of a limit function

$$
\varphi_{f}^{n}=\lim _{k \rightarrow \infty} \varphi_{k}^{n}
$$


on the set $B_{n}$, which is the bounded solution of the equation (2). Moreover, the function $\varphi_{f}^{n}$ is a continuation of the function $\varphi_{f}^{n-1}$ on the set $B_{n}$, i.e., $\left.\varphi_{f}^{n}\right|_{B_{n-1}}=\varphi_{f}^{n-1}$, and $\left|\varphi_{f}^{n}\right| \leqslant C$ for any $n$.

Next, consider the function

$$
\varphi_{f}=\left\{\begin{array}{l}
\varphi_{f}^{1} \quad \text { in } B_{1} \\
\varphi_{f}^{2} \quad \text { in } B_{2} \backslash B_{1} \\
\ldots \ldots \ldots \ldots \ldots \ldots \\
\varphi_{f}^{n} \text { in } B_{n} \backslash B_{n-1} \\
\ldots \ldots \ldots \ldots \ldots
\end{array}\right.
$$

Now choose the diagonal sequence $\varphi_{1}^{1}, \varphi_{2}^{2}, \ldots, \varphi_{k}^{k}, \ldots$ It is clear that $\left|\varphi_{k}^{k}\right| \leqslant C$, i. e., the diagonal sequence is uniformly bounded on $M$ and converges to the function $\varphi_{f}$ at each point $x \in M$. The uniform boundedness implies the compactness of the family of functions $\left\{\varphi_{k}^{k}\right\}$ in the class $C^{2}(G)$ on an arbitrary compact subset of $G \subset M$. The latter implies the existence of a limit function for this sequence, which is the solution of the equation (2) on $G$. Due to the uniqueness of the existence of the limit function, it coincides with the function $\varphi_{f}$. Thus, the $\varphi_{f}$ function is the solution of the equation (2) on $M$ and $\left|\varphi_{f}\right| \leqslant C$. The lemma is proved. $\square$

A similar approximative approach to determining solutions to boundaryvalue problems for elliptic equations on manifolds has been used earlier [15].

The following definitions and auxiliary statements from [14] will play a fundamental role in obtaining the results of this paper. We present them below. Let $f_{1}$ and $f_{2}$ be arbitrary continuous functions on $M$.

Definition 1. [14] Say that $f_{1}$ and $f_{2}$ are equivalent on $M$ and write $f_{1} \stackrel{M}{\sim} f_{2}$ if for some exhaustion $\left\{B_{k}\right\}_{k=1}^{\infty}$ of $M$ we have

$$
\lim _{k \rightarrow \infty}\left\|f_{1}(x)-f_{2}(x)\right\|_{C^{0}\left(M \backslash B_{k}\right)}=0,
$$

where $\|f(x)\|_{C^{0}(G)}=\sup _{G}|f(x)|$.

It is easy to verify that the relation $\stackrel{M}{\sim}$ is an equivalence relation, which does not depend on the choice of the exhaustion of the manifold and so partitions the set of all continuous functions on $M$ into equivalence classes (see also [12]). Denote the equivalence class of a function $f$ by $[f]$. 
Definition 2. [14] We call a function $\omega$ asymptotically nonnegative on $M$ if there exists a continuous function $f \geqslant 0$ on $M$, such that $\omega \stackrel{M}{\sim} f$.

Let $B \subset M$ be an arbitrary connected compact subset with a smooth boundary and $B \subset B_{k}$ for all $k$. Everywhere in the sequel we assume that $\left\{B_{k}\right\}_{k=1}^{\infty}$ is the exhaustion of the manifold $M$ with smooth boundaries $\partial B_{k}$.

Denote by $v_{k}$ the solution of the equation (1) in $B_{k} \backslash B$, satisfying the conditions

$$
\left.v_{k}\right|_{\partial B}=1,\left.\quad v_{k}\right|_{\partial B_{k}}=0 .
$$

In [14], it is shown that the sequence $v_{k}$ is uniformly bounded on $M \backslash B$ and, therefore, the sequence is compact in the class $C^{2}(G)$ for every compact subset $G \subset M \backslash B$. Moreover, as $k \rightarrow \infty$, this sequence increases monotonically and converges on $M \backslash B$ to a solution of equation (1) $v=\lim _{k \rightarrow \infty} v_{k}, \quad 0<v \leqslant 1,\left.\quad v\right|_{\partial B}=1$. Note, also, that the function $v$ does not depend on the choice of exhaustion $\left\{B_{k}\right\}_{k=1}^{\infty}$. We call $v$ the $L$-potential of the compact set $B$ relative to $M$. For the Laplace-Beltrami equation, the function $v$ is nothing else but the capacity potential of the compact set $B$ relative to the manifold $M$.

Definition 3. [14] Call the manifold $M$ L-strict if for some compact set $B \subset M$ there is an $L$-potential $v$ of $B$, such that $v \in[0]$ (if $L=\Delta$; then call the manifold $M \Delta$-strict).

Using the described approach, a number of statements, presented below, for solutions and super solutions of the stationary Schrödinger equation were obtained in [14]. These statements play an essential role in obtaining the main and auxiliary results of this paper.

Lemma 1. [14] Suppose that $L \omega \leqslant 0$ on $M \backslash B,\left.\omega\right|_{\partial B} \geqslant 0$, and $\omega$ is asymptotically nonnegative. Then $\omega \geqslant 0$ on $M \backslash B$.

Suppose that $L \omega \leqslant 0$ on $M$, and $\omega$ is asymptotically nonnegative. Then $\omega \geqslant 0$ on $M$.

Lemma 2. [14] Suppose that $L \omega \leqslant L u$ on $M \backslash B,\left.\omega\right|_{\partial B} \geqslant\left. u\right|_{\partial B}$, and $\omega \stackrel{M}{\sim} u$. Then $\omega \geqslant u$ on $M \backslash B$. In particular, if $L \omega=L u$ on $M \backslash B$, $\left.\omega\right|_{\partial B}=\left.u\right|_{\partial B}$, and $\omega \stackrel{M}{\sim} u$, then $\omega=u$ on $M \backslash B$.

Lemma 3. [14] Suppose that $L \omega \leqslant L u$ on $M$ and $\omega \stackrel{M}{\sim} u$. Then $\omega \geqslant u$ on $M$. In particular, if $L \omega=L u$ on $M$ and $\omega \stackrel{M}{\sim} u$, then $\omega=u$ on $M$. 
The paper [14] introduces the concepts of solvability of the boundaryvalue and external boundary-value problems for the homogeneous Schrödinger equation with boundary conditions from the equivalence class $[f]$. In a similar way, we can formulate boundary-value problems for the inhomogeneous equation (2) (see also [12]).

Definition 4. [12] We say that the boundary-value problem for equation (2) is solvable on $M$ with boundary data from the class $[f]$ if (2) has a solution $u \in[f]$ on $M$.

Definition 5. [12] Let $\Phi(x) \in C(\partial B)$ be any continuous function on $\partial B$. We say that the exterior boundary problem for equation (2) is solvable on $M \backslash B$ with boundary data from the class $[f]$ if for any function $\Phi(x) \in C(\partial B)$ equation (2) has a solution $u(x)$ on $M \backslash B$, such that $u \in[f]$ and $\left.u\right|_{\partial B}=\left.\Phi\right|_{\partial B}$.

The approach based on introduction of classes of equivalent functions allows to formulate boundary and exterior boundary-value problems on arbitrary noncompact Riemannian manifolds without taking into account their geometric compactification. Using this approach, the following relationship between the solvability of boundary-value and exterior boundary problems on the manifold $M$ was established in [12]:

Theorem 1. [12] Let the exterior boundary problem for equation (2) be solvable on $M \backslash B$ with boundary data $\left.u\right|_{\partial B}=\Phi(x)$ and $u \in[f]$ for any continuous function $\Phi(x) \in C(\partial B)$. Then the boundary-value problem for equation (2) with boundary data from the class $[f]$ is solvable on $M$ too.

Theorem 2. [12] Let $M$ be an L-strict manifold and the boundary-value problem for equation (2) be solvable on $M$ with boundary data from the class $[f]$. Then the exterior boundary problem for equation (2) is solvable on $M \backslash B$ with boundary data $\left.u\right|_{\partial B}=\Phi(x)$ and $u \in[f]$ for any continuous function $\Phi(x) \in C(\partial B)$.

Remark 2. Similar results for the stationary Schrödinger equation can be found, for example, in [8] and [14].

It is known that any constant, in particular, the function $u \equiv 1$, is a harmonic function, i.e., it satisfies the equation (1) for $c(x) \equiv 0$ on any manifold $M$. In this case, $u \in[1]$. However, for the stationary Schrödinger equation, where $c(x) \not \equiv 0$, a nonzero constant is not a solution. It is known that the existence of the solution $u \in[1]$ on $M$ of the equation (1), where $c(x) \not \equiv 0$, depends on the properties of the noncompact manifold $M$ (see, 
for example, [10]). For manifolds, on which such solutions exist, a special definition has been introduced in [14].

Definition 6. [14] Say that a manifold $M$ is $L$-exact if there is a solution $u$ to equation (1) on $M$, satisfying the condition $u \in[1]$.

Further, alongside with the Schrödinger operator $L$, we consider the Schrödinger operator

$$
L_{1} \equiv \Delta-c_{1}(x)
$$

where $0 \leqslant c_{1}(x) \leqslant c(x), c_{1}(x) \not \equiv 0$, and $c_{1}(x) \in C^{0, \alpha}(G)$ for all precompact subset $G \subset M, 0<\alpha<1$. The following statement is true:

Lemma 4. If the manifold $M$ is $L$-exact, then the manifold $M$ is $L_{1}$-exact, i. e., there exists a solution $u_{1}$ of the equation $L_{1} u=0$ on $M$, such that $u_{1} \in[1]$.

Proof. By the condition of the lemma, there exists a solution $u$ of the equation (1) on $M$, such that $u \in[1]$. By Lemma 1, the inequality $u \geqslant 0$ holds on $M$. Consider a sequence of functions $\left\{w_{k}\right\}_{k=1}^{\infty}$ in $B_{k}$ that are the solutions to the problems

$$
\left\{\begin{array}{l}
L_{1} w_{k}=0 \quad \text { in } \quad B_{k} \\
\left.w_{k}\right|_{\partial B_{k}}=1
\end{array}\right.
$$

Denote $w_{k}{ }^{*}=w_{k}-1$ in $B_{k}$. Then $L_{1} w_{k}{ }^{*}=L_{1} w_{k}-L_{1} 1=c_{1}(x) \geqslant 0$ in $B_{k}$ and $\left.w_{k}{ }^{*}\right|_{\partial B_{k}}=\left.w_{k}\right|_{\partial B_{k}}-1=0$. Then, using the comparison principle for solutions of the Schrödinger equation in $B_{k}$, we get $w_{k}{ }^{*} \leqslant 0$ in $B_{k}$. It follows that $0 \leqslant w_{k} \leqslant 1$ in $B_{k}$, for any $k$, i. e., the sequence of solutions $\left\{w_{k}\right\}_{k=1}^{\infty}$ is uniformly bounded on $M$.

The uniform boundedness of the sequence $\left\{w_{k}\right\}_{k=1}^{\infty}$ on $M$ implies the compactness of the family of functions $\left\{w_{k}\right\}$ in the class $C^{2}(G)$ for an arbitrary compact subset $G \subset M$, by the Compactness lemma. Next, by the Existence lemma for the family $\left\{w_{k}\right\}$, we obtain the existence of a limit function $u_{1}$, which is the solution of the equation $L_{1} u_{1}=0$ on $M$ and satisfies the inequality $0 \leqslant u_{1} \leqslant 1$.

In addition, the following conditions hold in $B_{k}$ for the sequence of functions $\left\{w_{k}\right\}_{k=1}^{\infty}$ :

$$
\left\{\begin{array}{l}
L w_{k} \leqslant L u \text { in } \quad B_{k}, \\
\left.w_{k}\right|_{\partial B_{k}} \geqslant\left. u\right|_{\partial B_{k}} .
\end{array}\right.
$$


Then, according to the comparison principle for solutions of the Schrödinger equation in $B_{k}$, we have $w_{k} \geqslant u$ and, consequently, $1 \geqslant u_{1} \geqslant u$. Given $u \in[1]$, we get $u_{1} \in[1]$, i. e., the manifold $M$ is $L_{1}$-exact.

3. The main results. The following theorem establishes the relationship between solvability of boundary-value problems in a given equivalence class for the inhomogeneous Schrödinger equation (2) with some variations of its coefficient $c(x)$, and generalizes the result obtained earlier in [14] for the homogeneous Schrödinger equation (1) for the inhomogeneous case.

Theorem 3. Suppose that the boundary-value problems with boundary data in the class $[f]$ are solvable on $M$ for the inhomogeneous equations $L u=g(x)$ and $\Delta u=g(x)$ on $L$-exact manifold $M$. Then the boundary-value problem is solvable on $M$ for the inhomogeneous equation $L_{1} u=g(x)$ with boundary data in the class $[f]$.

Proof. Let $v$ and $w$ be bounded solutions on $M$ to the boundary-value problems for the equations $L u=g(x)$ and $\Delta u=g(x)$, respectively, such that $v \in[f], w \in[f]$. Since $f$ is a bounded continuous function on $M$, the solutions $v$ and $w$ are also bounded on $M$, i. e., the inequalities are satisfied: $|f(x)| \leqslant A,|v(x)| \leqslant A$ and $|w(x)| \leqslant A$, where $A>0$ is a constant. Also, let $u$ be the solution of the stationary Schrödinger equation, i. e., $u \in[1]$ (it exists because the manifold is $L$-exact).

Consider the functions $f_{A}=f+A, v_{A}=v+A \cdot u, w_{A}=w+A$. It is clear that all functions are non-negative: $f_{A} \geqslant 0, v_{A} \geqslant 0, w_{A} \geqslant 0$; that $v_{A} \in\left[f_{A}\right], w_{A} \in\left[f_{A}\right]$; and that $L v_{A}=L v+A \cdot L u=g(x)$, $\Delta w_{A}=\Delta w=g(x)$. We show that the theorem is true in the class of nonnegative functions $f_{A}$.

As before, let $\left\{B_{k}\right\}_{k=1}^{\infty}$ be an exhaustion of $M$ with smooth boundaries $\partial B_{k}$. Consider the sequence of functions $\left\{u_{k}\right\}_{k=1}^{\infty}$ that are solutions to the problems

$$
\left\{\begin{array}{l}
L_{1} u_{k}=g(x) \text { in } B_{k}, \\
\left.u_{k}\right|_{\partial B_{k}}=\left.w_{A}\right|_{\partial B_{k}} .
\end{array}\right.
$$

Denote by $u_{k}{ }^{*}=u_{k}-w_{A}$ in $B_{k}$. Then we have

$$
L_{1} u_{k}^{*}=L_{1} u_{k}-L_{1} w_{A}=g(x)-\Delta w_{A}+c_{1}(x) w_{A}=c_{1}(x) w_{A}
$$

and $\left.u_{k}{ }^{*}\right|_{\partial B_{k}}=\left.u_{k}\right|_{\partial B_{k}}-\left.w_{A}\right|_{\partial B_{k}}=0$. Since $c_{1}(x) w_{A} \geqslant 0$ in $B_{k}, L_{1} u_{k}{ }^{*} \geqslant 0$ in $B_{k}$ and $\left.u_{k}{ }^{*}\right|_{\partial B_{k}}=0$. Then, by the comparison principle (see [2, pp. 3940]), we have $u_{k}{ }^{*} \leqslant 0$ in $B_{k}$. It follows that

$$
u_{k} \leqslant w_{A} \quad \text { in } \quad B_{k} \text {. }
$$


Further, consider the sequence of functions $\left\{v_{k}\right\}_{k=1}^{\infty}$ that are solutions to the problems

$$
\left\{\begin{array}{l}
L_{1} v_{k}=g(x) \quad \text { in } \quad B_{k}, \\
\left.v_{k}\right|_{\partial B_{k}}=\left.v_{A}\right|_{\partial B_{k}} .
\end{array}\right.
$$

Denote by $v_{k}{ }^{*}=v_{k}-v_{A}$ in $B_{k}$. As above, we have

$L_{1} v_{k}{ }^{*}=L_{1} v_{k}-L_{1} v_{A}=g(x)-\Delta v_{A}+c_{1}(x) v_{A} \leqslant g(x)-\Delta v_{A}+c(x) v_{A}=0$,

$\left.v_{k}^{*}\right|_{\partial B_{k}}=\left.v_{k}\right|_{\partial B_{k}}-\left.v_{A}\right|_{\partial B_{k}}=0$. Hence, $L_{1} v_{k}^{*} \leqslant 0$ in $B_{k}$ and $\left.v_{k}^{*}\right|_{\partial B_{k}}=0$. Moreover, by the comparison principle (see [2, pp. 39-40]), we obtain $v_{k}{ }^{*} \geqslant 0$ in $B_{k}$ and, therefore,

$$
v_{k} \geqslant v_{A} \quad \text { in } \quad B_{k}
$$

Compare solutions $v_{A}$ and $w_{A}$. By the condition of the theorem, it is fulfilled on $M: L v_{A}=g(x)$ and $\Delta w_{A}=g(x)$. Then, for the operator $L$ we have

$$
L w_{A}=\Delta w_{A}-c(x) w_{A} \leqslant \Delta w_{A}=g(x),
$$

since $c(x) w_{A} \geqslant 0$. It follows that $L w_{A} \leqslant g(x)$ on $M$. Further, given that $L v_{A}=g(x)$, we obtain $L w_{A} \leqslant L v_{A}$ on $M$ and $w_{A} \stackrel{M}{\sim} v_{A} \stackrel{M}{\sim} f_{A}$. So, applying Lemma 3, we have $w_{A} \geqslant v_{A}$ on $M$.

Now we compare solutions $u_{k}$ and $v_{k}$. From conditions (3), (5) we have the equality $L_{1} u_{k}=L_{1} v_{k}$ in $B_{k}$, and, therefore, $L_{1}\left(u_{k}-v_{k}\right)=0$. Assuming that $w_{A} \geqslant v_{A}$ on $M$, we obtain $\left.u_{k}\right|_{\partial B_{k}} \geqslant\left. v_{k}\right|_{\partial B_{k}}$. Then, by the comparison principle for the solution of the Schrödinger equation (see [2, pp. 39-40]), we have

$$
u_{k} \geqslant v_{k} \text { in } B_{k} .
$$

Combining the conditions (4), (6), and (7), gives the following inequality for all $k$ in $B_{k}$ :

$$
w_{A} \geqslant u_{k} \geqslant v_{k} \geqslant v_{A} \geqslant 0
$$

Since the functions $v_{A}$ and $w_{A}$ are bounded solutions of the corresponding equations on $M$, condition (8) implies the uniform boundedness of the family of functions $\left\{u_{k}\right\}_{k=1}^{\infty}$ and $\left\{v_{k}\right\}_{k=1}^{\infty}$ on $M$.

Uniform boundedness of the sequences $\left\{u_{k}\right\}_{k=1}^{\infty}$ and $\left\{v_{k}\right\}_{k=1}^{\infty}$ on $M$ implies, by the Compactness lemma, compactness of the families of functions $\left\{u_{k}\right\}$ and $\left\{v_{k}\right\}$ in the class $C^{2}(G)$ for an arbitrary compact subset $G \subset M$. 
In turn, according to the Existence lemma, the compactness of the families $\left\{u_{k}\right\}$ and $\left\{v_{k}\right\}$ implies the existence of limit functions $\bar{u}=\lim _{i \rightarrow \infty} u_{k_{i}}$ and $\underline{u}=\lim _{i \rightarrow \infty} v_{k_{i}}$, which are solutions of the equation $L_{1} u=g(x)$.

Passing to the limit in (8) as $k \rightarrow \infty$, we find that on $M$

$$
w_{A} \geqslant \bar{u} \geqslant \underline{u} \geqslant v_{A} \geqslant 0 .
$$

From (9) and the fact that $w_{A} \sim v_{A} \sim f_{A}$, we get $\bar{u} \stackrel{M}{\sim} \underline{u} \stackrel{M}{\sim} f_{A}$; so, according to Lemma 3 , we get $\bar{u}=\underline{u}=u_{A}$ and $u_{A} \geqslant 0$.

We have proved solvability of the boundary problem for equation $L_{1} u=g(x)$ with boundary data in the class $\left[f_{A}\right]$ and function $u_{A}$ is the corresponding solution.

Then, by Lemma 4 , we get its $L_{1}$-exactness from the $L$-exactness of the manifold. Finally, consider the function $u=u_{A}-A \cdot u_{1}$, where $u_{1}$ is the function from the definition of $L_{1}$-exactness. It is obvious that the following equalities are fulfilled on $M$ :

$$
L_{1} u=L_{1} u_{A}-A \cdot L_{1} u_{1}=g(x), \quad u \stackrel{M}{\sim} f_{A}-A=f+A-A=f .
$$

Thus, we have solvability of the boundary problem for equation $L_{1} u=g(x)$ on $M$ with boundary data in the class $[f]$.

The next theorem establishes relationship between solvability of exterior boundary-value problems on $M \backslash B$ in a given equivalence class for the inhomogeneous Schrödinger equation (2) with some variations of its coefficient $c(x)$. As above, here $B \subset M$ is any precompact subset with smooth boundary; also assume that $0 \leqslant c_{1}(x) \leqslant c(x), c_{1}(x) \not \equiv 0$ on $M$, and so $c(x) \not \equiv 0$ on $M$. Without loss of generality, we can assume that $c(x)>0$ in some neighborhood of the compact subset $B$.

Theorem 4. Suppose that the exterior boundary problems with boundary data in the class $[f]$ are solvable on $M \backslash B$ for the inhomogeneous equations $L u=g(x)$ and $\Delta u=g(x)$ on an $L$-exact manifold $M$. Then the exterior boundary problem is solvable on $M \backslash B$ for the inhomogeneous equation $L_{1} u=g(x)$ with boundary data in the class $[f]$.

Proof. It is shown in Theorem 1 (see also [12]) that solvability of exterior boundary-value problems on $M \backslash B$ with boundary data in the class $[f]$ for the inhomogeneous equations $L u=g(x)$ and $\Delta u=g(x)$ implies solvability of boundary-value problems for these equations on $M$ with 
the same asymptotic boundary data. Further, from Theorem 3 solvability of boundary-value problems on $M$ for the inhomogeneous equation $L_{1} u=g(x)$ with boundary data in the class $[f]$ follows. Now it suffices to show that under the conditions of Theorem 4 the manifold $M$ is $L_{1}$-strict, i. e., there is an $L_{1}$-potential $v_{L_{1}}$ of the compact set $B$, such that $v_{L_{1}} \in[0]$ (see Theorem 2 or [12]).

Consider some sequence of functions $v_{k}$ that are solutions of homogeneous Schrödinger equation $L_{1} v_{k}=0$ in $B_{k} \backslash B$ and satisfy the conditions

$$
\left.v_{k}\right|_{\partial B}=1,\left.\quad v_{k}\right|_{\partial B_{k}}=0 .
$$

As above, the sequence $v_{k}$ is uniformly bounded and monotone on $M \backslash B$, hence, it converges on $M \backslash B$ to the function $v_{L_{1}}=\lim _{k \rightarrow \infty} v_{k}$, such that

$$
L_{1} v_{L_{1}}=0, \quad 0<v_{L_{1}} \leqslant 1,\left.\quad v_{L_{1}}\right|_{\partial B}=1 .
$$

By definition, the function $v_{L_{1}}$ is an $L_{1}$-potential of the compact set $B$ relatively to the manifold $M$. Let us show that $v_{L_{1}} \in[0]$.

By the condition of the theorem, the exterior boundary-value problems for the Poisson equation $\Delta u=g(x)$ are solvable on $M \backslash B$ with boundary data in the class $[f]$; that is, there exist solutions $u_{1}$ and $u_{2}$ of this equation, such that $\left.u_{1}\right|_{\partial B}=1,\left.u_{2}\right|_{\partial B}=2$, and $u_{1} \in[f], u_{2} \in[f]$. Consider the difference $u=u_{2}-u_{1}$. It is clear that the function $u$ is harmonic on $M \backslash B$, satisfies the boundary conditions $\left.u\right|_{\partial B}=1$ and $u \in[0]$, as well as the inequality $0<u \leqslant 1$ due to the maximum principle.

From the definition of the function $v_{k}$ in $B_{k} \backslash B$, we have

$$
\Delta v_{k}=L_{1} v_{k}+c_{1} v_{k}=c_{1}(x) v_{k} \geqslant 0,
$$

i. e., for each $k$ the function $v_{k}$ is subharmonic. Moreover, $v_{k}$ satisfies the relations $\left.v_{k}\right|_{\partial B}=\left.u\right|_{\partial B}$ and $\left.v_{k}\right|_{\partial B_{k}} \leqslant\left. u\right|_{\partial B_{k}}$. Then, applying the comparison principle for harmonic functions in $B_{k} \backslash B$, we obtain $0 \leqslant v_{k} \leqslant u$ for any $k$. Passing to the limit as $k \rightarrow \infty$, we get $0<v_{L_{1}} \leqslant u$. Taking into account that $u \in[0]$, we conclude that $v_{L_{1}} \in[0]$, i. e, $M$ is an $L_{1}$-strict manifold.

Thus, from solvability of the boundary-value problem on the manifold $M$ for the equation $L_{1} u=g(x)$ with boundary data in the class $[f]$ and the $L_{1}$-strictness of the manifold, we obtain the solvability on $M \backslash B$ of the exterior boundary-value problem for the equation $L_{1} u=g(x)$ with boundary data in the class $[f]$ due to Theorem 2 (see also [12]). The theorem is proved. 
Boundary-value problems for the inhomogeneous Schrödinger equation127

Acknowledgments. This work was partially supported by the Ministry of Science and Higher Education of the Russian Federation (government task no. 0633-2020-0003).

\section{References}

[1] Anderson M. T. The Dirichlet problem at infinity for manifolds with negative curvature. J. Differential Geom., 1983, vol. 18, no. 4, pp. 701-721. DOI: https://doi.org/10.4310/jdg/1214438178

[2] Gilbarg D. and Trudinger N. S. Elliptic partial differential equations of second order. Springer-Verlag, Berlin, Heidelberg, New York, Tokyo, 1983.

[3] Grigor'yan A. Analitic and geometric background of recurence and nonexplosion of the Brownian motion on Riemannian manifolds. Bull. Amer. Math. Soc., 1999, vol. 36, pp. 135-249.

[4] Grigor'yan A. A., Losev A. G. Dimension of spaces of solutions of the Schrödinger equation on noncompact Riemannian manifolds. Mathematical physics and computer simulation, 2017, vol. 20, no. 3, pp. 34-42 [in Russian]. DOI: https://doi.org/10.15688/mpcm.jvolsu.2017.3.3

[5] Grigor'yan A. A., Nadirashvili N. S. Liouville theorems and external boundary value problems. Russian Math. (Iz. VUZ), 1987, no. 5, pp. 25-33.

[6] Kesel'man V. M. The concept and criteria of the capacitive type of the noncompact Riemannian manifold based on the Generalized capacity. Mathematical physics and computer simulation, 2019, vol. 22, no. 2, pp. 21-32 [in Russian]. DOI: https://doi.org/10.15688/mpcm.jvolsu.2019.2.2

[7] Korolkov S. A. Harmonic functions on Riemannian manifolds with ends. Sib. Math. J., 2008, vol. 49, no. 6, pp. 1319-1332.

DOI: https://doi.org/10.1007/s11202-008-0101-1

[8] Korol'kov S. A. On the solvability of boundary value problems for the stationary Schrödinger equation in unbounded domains on Riemannian manifolds. Differ. Equ., 2015, vol. 51, no. 6, pp. $738-744$.

DOI: https://doi.org/10.1134/S001226611506004X

[9] Losev A. G. On the solvability of the Dirichlet problem for the Poisson equation on certain noncompact Riemannian manifolds. Differ. Equ., 2017, vol. 53. no. 12 , pp. $1643-1652$.

DOI: https://doi.org/10.1134/S0012266117120072

[10] Losev A. G., Mazepa E. A. Bounded solutions of the Schrödinger equation on Riemannian products St. Petersburg Math. J., 2002, vol. 13, no. 1, pp. $57-73$. 
[11] Losev A.G., Mazepa E.A., Chebanenko V.Y. On unbounded solutions of the stationary Schrödinger equation on model Riemannian manifolds. Russian Math. (Iz. VUZ), 2006, no. 7, pp. 46-64.

DOI: https://doi.org/10.1007/BF03321048

[12] Losev A. G., Mazepa E. A. On solvability of the boundary value problems for the inhomogeneous elliptic equations on noncompact Riemannian manifolds. Probl. Anal. Issues Anal., 2018, vol. 7, no. 25, Special Issue, pp. 101-112. DOI: https://doi.org/10.15393/j3.art.2018.5330

[13] Mastrolia P., Monticelli D. D., Punzo F. Elliptic and parabolic equations with Dirichlet conditions at infinity on Riemannian manifolds. Adv. Differential Equations, 2018, vol. 23, no. 1/2, pp. 89-108.

[14] Mazepa E. A. Boundary value problems for stationary Schrödinger equation on Riemannian manifolds Sib. Math. J., 2002, vol. 43, no. 3, pp. 591-599. DOI: https://doi.org/10.1023/A:1015411502059

[15] Mazepa E. A. An approximate approach to the construction of solutions to boundary value problems on non-compact Riemannian manifolds. Vestn. Volgogr. Univ. Mat. Fiz., 2015, vol. 30, no. 5, pp. $25-35$ [in Russian]. DOI: http://dx.doi.org/10.15688/jvolsu1.2015.5.2

[16] Munteanu O., Sesum N. The Poisson equation on complete manifolds with positive spectrum and applications. Adv. in Math., 2010, vol. 223, pp. 198-219. DOI: https://doi.org/10.1016/j.aim.2009.08.003

[17] Murata M. Positive harmonic functions on rotationary symmetric Riemannian manifolds in: Potential Theory (Proc. Intern. Conf. Nagoya/Japan, 1990), 1992, pp. $251-259$.

DOI: https://doi.org/10.1515/9783110859065.251

[18] Sullivan D. The Dirichlet problem at infinity for a negatively curved manifold. J. Differential Geom., 1983, vol. 18, no. 4, pp. 723-732.

DOI: https://doi.org/10.4310/jdg/1214438179

Received June 19, 2021.

In revised form, October 12, 2021.

Accepted October 15, 2021.

Published online October 27, 2021.

Volgograd State University

100 Universitetsky pr., Volgograd 400062, Russia

E. A. Mazepa elena.mazepa@volsu.ru

D. K. Ryaboshlykova daria_ryaboshlikova@volsu.ru 